This item was submitted to Loughborough's Research Repository by the author.

Items in Figshare are protected by copyright, with all rights reserved, unless otherwise indicated.

\title{
Outage probability analysis for two-antennas MISO-NOMA downlink with statistical CSI
}

PLEASE CITE THE PUBLISHED VERSION

https://doi.org/10.1109/GLOBECOM38437.2019.9014301

PUBLISHER

IEEE

VERSION

AM (Accepted Manuscript)

\section{PUBLISHER STATEMENT}

(c) 2019 IEEE. Personal use of this material is permitted. Permission from IEEE must be obtained for all other uses, in any current or future media, including reprinting/republishing this material for advertising or promotional purposes, creating new collective works, for resale or redistribution to servers or lists, or reuse of any copyrighted component of this work in other works.

\section{LICENCE}

\section{All Rights Reserved}

\section{REPOSITORY RECORD}

Li, Shenhong, Mahsa Derakhshani, Chung Shue Chen, and Sangarapillai Lambotharan. 2020. "Outage Probability Analysis for Two-antennas MISO-NOMA Downlink with Statistical CSI". figshare. https://hdl.handle.net/2134/9929783.v1. 


\section{Outage Probability Analysis for Two-antennas MISO-NOMA Downlink with Statistical CSI}

\author{
Shenhong Li \\ Wolfson School \\ Loughborough University \\ Loughborough, UK \\ S.Li3@lboro.ac.uk
}

\author{
Mahsa Derakhshani \\ Wolfson School \\ Loughborough University \\ Loughborough, UK \\ M.Derakhshani@lboro.ac.uk
}

\author{
Chung Shue Chen \\ Bell Labs, Nokia Paris-Saclay \\ Nozay, France \\ Chung_Shue.Chen \\ @ nokia-bell-labs.com
}

\author{
Sangarapillai Lambotharan \\ Wolfson School \\ Loughborough University \\ Loughborough, UK \\ S.Lambotharan@lboro.ac.uk
}

\begin{abstract}
In this paper, we analyze the outage probability of the multi-user multiple-input single-output (MISO) downlink system by combining the non-orthogonal multiple access (NOMA) scheme. We derive tractable closed-form outage expressions given a minimum target rate for the individual users for the case of two antennas, by modeling cumulative distribution function (CDF) of received signal-to interference plus noise ratio (SINR). Simulation results illustrate the outage performance for different power allocation scenarios and verify the accuracy of our outage probability analysis.
\end{abstract}

Index Terms-NOMA, MISO, Outage probability.

\section{INTRODUCTION}

Currently, non-orthogonal multiple access (NOMA) has received significant attention for future generation networks that can achieve low latency, massive connectivity, high reliability, and high throughput [1]. Multiple-input and singleoutput (MISO) [2] is one of the smart antenna technologies for wireless communications in which antennas are used at the transmitter side, and the received side only has one antenna. From the application viewpoint, the combination of NOMA and multiple antenna techniques is effective to enhance system performance. The essential intention of NOMA scheme in this work is to simultaneously serve multiple users over the same radio resources by applying successive interference cancellation (SIC) at the receiver side to cancel the inter-user interference.

Zero-forcing beamforming (ZFBF) is a practical multi-user transmission strategy. By designing one user's beamforming weight vector to be orthogonal to other selected user's channel vectors, ZFBF can completely eliminate multiuser interference [3]. Unlike conventional multi-user beamforming, NOMAbased beamforming involves designing a single beamforming vector to support multiple users. Hence, analyzing the MISONOMA systems becomes a prominent research trend. In terms of system performance, the authors in [4] demonstrated that the sum rate of MIMO-NOMA system is greater than that of MIMO-OMA system. Moreover, in order to further improve the system performance, the authors in [5] studied the sum rate maximization problem for MISO-NOMA downlink

This work has been supported by the Engineering and Physical Sciences Research Council through grant EP/R006385/1. A part of the work was carried out at LINCS (www.lincs.fr) system and proposed an iterative algorithm to solve a secondorder cone program (SOCP) with polynomial computational complexity. [6] examined iterative user clustering, and joint optimization with beamforming design and power allocation for MISO-NOMA downlink systems. The authors in [7] investigated the ergodic rate maximization problem for MISONOMA system considering statistical CSI under a constraint on the total transmission power. On the other hand, the authors in [8] proposed a minimum total power problem in MISO-NOMA system. A dynamic clustering optimization problem from a fairness perspective was investigated in [9] for MIMO-NOMA systems. Since outage probability is another evaluation criterion for system performance, it attracts many researchers to study it.

In conventional NOMA systems, the outage probability is widely used in the existing works [10], [11] and [12] for performance analyses when the channel is characterized by random parameters. Furthermore, for MIMO-NOMA systems, the rate outage probability has been analyzed in [13] considering imperfect scenario, where channel gains are modelled as the estimated values plus an estimation error. Moreover, the outage probability expression in the case of channel estimation error is approximated by applying central limit theorem (CLT) in the case of a large number of antennas.

In this paper, the downlink of single cell MISO-NOMA system with one multi-antenna base station serving singleantenna users is considered where users are grouped to multiple clusters. NOMA is implemented for the users within each cluster, while ZFBF is used to cancel inter-cluster interference. We also assume that maximum two users can be in one cluster, to keep the SIC complexity low, which is a common assumption in the NOMA literature. We concentrate on the analytical study of the behavior of outage probability for individual users. Specifically, we derive a tractable closedform outage expression, which is defined as the probability that the instantaneous data rate is below a target value, for the case of two antennas. The outage probability of the users with more favorable channel conditions (called near users) can be obtained based on the cumulative distribution function (CDF) of exponential distribution for Rayleigh channels. On the other hand, the analysis of outage probability of the users with less favorable channel conditions (far users) is more challenging. 
We derive approximated closed form expressions for far users based on the CDF of the ratio between two exponentially distributed random variables. This work only focuses on the case of two antennas at the BS since it is the first step to provide insights on rate outage analysis for MISO-NOMA systems under statistical CSI and as far as we know it has not been investigated in the MISO-NOMA literature previously. This analysis will be extended for a general case with $N$ antennas in the future works.

The rest of this paper is organized as follows. Section II outlines the system model. Section III introduces the techniques of beamforming design and NOMA scheme. In Section IV, we formulate the received signal to interference plus noise ratio (SINR) of each user. Then, we analyse the outage probability of near and far users in Section V. Simulation results are shown in Section VI, followed by conclusions in Section VII.

We use boldface capital and lower case letters to denote matrices and vectors, respectively. (. $)^{T}$ to denote the transpose, $\mathbf{E}($.$) to denotes the expectation.$

\section{SYSTEM MODEL}

Consider a multiuser downlink MISO system consisting of one base station (BS) with two antennas, serving two clusters of users, each equipped with single receive antennas. The belonging users to one cluster share a beamforming, and thus, NOMA is implemented as the multiple access technique in each cluster. As shown in Fig. 1, we further assume that each cluster has only two users to keep the SIC complexity low. Based on the distance from BS, the two users in each cluster are called either near and far users.

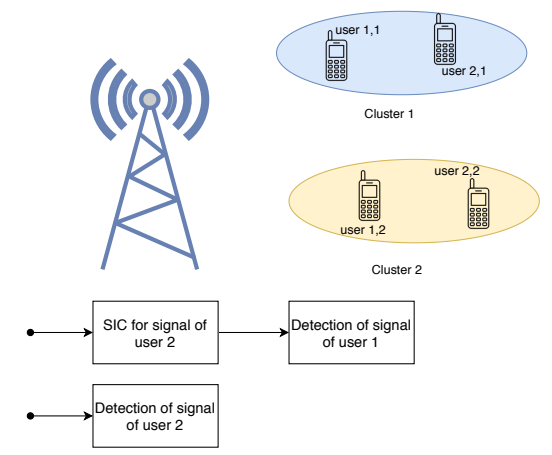

Fig. 1: Downlink MISO-NOMA system

In practice, it is difficult to acquire the accurate channel state information due to channel estimation and quantization. In this work, we assume that only the statistical knowledge of CSI is available, hence the channel (row) vector for each user at cluster $n$ is modeled as a random vector $\mathbf{h}_{i, n}=$ $\left[h_{i, n}^{m}\right] \in \mathbb{C}^{1 \times 2}$, where $i$ represents the index for users, $n$ denotes the index for clusters, and $m$ represents the index for antennas. All of them can be equal to either 1 or 2 . Assuming all wireless channels in the network exhibit Rayleigh block flat fading characteristics, the entries in $\mathbf{h}_{i, n}$ can be modeled as independent and identically distributed (i.i.d.) complex Gaussian random variables with zero-mean and unit variance.
We adopt linear transmit precoding at the BS, where each cluster is assigned with one dedicated information beam. The weights for all antennas for the near user at cluster $n$ can be collected into a $2 \times 1$ beamforming vector $\mathbf{w}_{n}=\left[w_{1, n}, w_{2, n}\right]^{T}$, where (. $)^{T}$ denotes the transpose of vector. Accordingly, the received signals in the cluster $n$ for two users are given by

$$
\begin{gathered}
y_{i, n}=\mathbf{h}_{i, n} \sqrt{d_{i, n}^{-\lambda}} \sum_{j=1}^{2} \sqrt{P_{j}} \mathbf{w}_{j} x_{j}+e_{i, n}, \\
(i=1,2 ; n=1,2)
\end{gathered}
$$

where the transmitted signal $x_{j}$ is expressed as the scalar value $x_{j}=\sqrt{\alpha_{1, j}} s_{1, j}+\sqrt{\alpha_{2, j}} s_{2, j}$, in which $\alpha_{1, j}$ and $\alpha_{2, j}$ are the transmit power allocation coefficients for two users in cluster $j$ such that $\alpha_{1, j}+\alpha_{2, j}=1 ; s_{1, j}$ and $s_{2, j}$ are the message signals for both users in cluster $j ; e_{i, n} \sim \mathcal{C N}\left(0, \sigma^{2}\right)$ denotes the i.i.d. complex Gaussian noise value. Moreover $P_{j}$ is the transmit power for cluster $j, d_{i, n}$ is the distance of user $i$ in cluster $n$ from the $\mathrm{BS}$, and $\lambda$ is the path loss exponent.

\section{PRoposed TeChNiques}

\section{A. Beamforming Design}

Transmit beamforming uses multiple antennas and channel state information at the transmitter (CSIT) to steer radiated power towards directions of interest, limiting leakage in other directions. The traditional approach for multi user MIMO processing is to derive the sum capacity by dirty paper coding (DPC) [14]. However, such coding scheme is difficult to implement in practical system because of brute-forcing method. The simple zero-forcing method is utilized in this work to cancel the inter-cluster interference. Unlike conventional multi-user beamforming, NOMA-based multiuser beamforming involves designing a single beamforming vector to support multiple users in a cluster. In this system, the channel gain vectors of users are proposed as

$$
\begin{array}{ll}
\mathbf{h}_{1,1}=\left[h_{1,1}^{1}, h_{1,1}^{2}\right], & \mathbf{h}_{2,1}=\left[h_{2,1}^{1}, h_{2,1}^{2}\right], \\
\mathbf{h}_{1,2}=\left[h_{1,2}^{1}, h_{1,2}^{2}\right], & \mathbf{h}_{2,2}=\left[h_{2,2}^{1}, h_{2,2}^{2}\right],
\end{array}
$$

where the superscript of $h_{1,1}^{1}$ is the index of antenna, the subscript $\{1,1\}$ represents the near user located at cluster 1 , and the subscript $\{2,1\}$ represents the far user at cluster 1. Thus, the beamforming vector $\mathbf{w}_{n}$ for cluster $n$ is chosen to be orthogonal to all other clusters' channel vector $\mathbf{h}_{1, n^{\prime}}$, satisfying the following condition

$$
\frac{\mathbf{h}_{1, n^{\prime}}}{\left|\mathbf{h}_{1, n^{\prime}}\right|} \mathbf{w}_{n}= \begin{cases}0, & \text { for } \quad n^{\prime} \neq n \\ 1, & \text { for } \quad n^{\prime}=n .\end{cases}
$$

The beamforming matrix in such MISO-NOMA system is designed to cancel out the inter-cluster interference for near users of all clusters, so that they can effectively perform SIC using NOMA.

When the normalized channel gain matrix for the near user is represented as

$$
\mathbf{H}_{1}=\left[\begin{array}{cc}
\frac{h_{1,1}^{1}}{\left|\mathbf{h}_{1,1}\right|} & \frac{h_{1,1}^{2}}{\left|\mathbf{h}_{1,1}\right|} \\
\frac{h_{1,2}^{1,}}{\left|\mathbf{h}_{1,2}\right|} & \frac{h_{1,2}^{2}}{\left|\mathbf{h}_{1,2}\right|}
\end{array}\right],
$$


where each antenna of channel gain follows the Rayleigh fading, the corresponding zero-beamforming matrix of the square channel gain matrix becomes

$$
\begin{aligned}
\mathbf{W} & =\mathbf{H}_{\mathbf{1}}{ }^{-1} \\
& =\frac{\left|\mathbf{h}_{1,1}\right|\left|\mathbf{h}_{1,2}\right|}{h_{1,1}^{1} h_{1,2}^{2}-h_{1,2}^{1} h_{1,1}^{2}}\left[\begin{array}{cc}
h_{1,2}^{2}\left|\mathbf{h}_{1,1}\right| & -h_{1,1}^{2}\left|\mathbf{h}_{1,2}\right| \\
-h_{1,2}^{1}\left|\mathbf{h}_{1,1}\right| & h_{1,1}^{1}\left|\mathbf{h}_{1,2}\right|
\end{array}\right] .
\end{aligned}
$$

\section{B. NOMA scheme}

In this work, NOMA is proposed to cancel the inter-user interference in each cluster. The SIC is utilized at the received side to separate signals in each cluster. However, it is hard to determine the decoding order since we only know the statistical knowledge of distribution of CSI at the transmitter. The SIC receiver first decodes the near user's signal by treating the far user's signal as the interference. After this detection stage, the near user's signal is removed from the composition signal. The far user treats the signal of the near user as interference without cancellation.

\section{RECEIVED SINR}

In this section, we derive the signal to interference plus noise ratio (SINR) of the near and far users for the proposed MISO-NOMA system. The received signal of the near user in cluster 1 can be formulated as

$$
\begin{aligned}
y_{1,1}= & \mathbf{h}_{1,1} \sqrt{d_{1,1}^{-\lambda} P_{1}} \mathbf{w}_{1} x_{1}+\mathbf{h}_{1,1} \sqrt{d_{1,1}^{-\lambda} P_{2}} \mathbf{w}_{2} x_{2}+e_{1,1} \\
= & \mathbf{h}_{1,1} \sqrt{d_{1,1}^{-\lambda} P_{1}} \mathbf{w}_{1}\left(\sqrt{\alpha_{1,1}} s_{1,1}+\sqrt{\alpha_{2,1}} s_{2,1}\right) \\
& +\mathbf{h}_{1,1} \sqrt{d_{1,1}^{-\lambda} P_{2}} \mathbf{w}_{2} x_{2}+e_{1,1},
\end{aligned}
$$

where $\mathbf{h}_{1,1} \sqrt{d_{1,1}^{-\lambda} P_{2}} \mathbf{w}_{2} x_{2}$ is the inter-cluster interference from the cluster 2 , which can be removed by using $\mathrm{ZF}$ beamforming method and $\mathbf{h}_{1,1} \sqrt{d_{1,1}^{-\lambda} P_{1}} \mathbf{w}_{1} \sqrt{\alpha_{2,1}} s_{2,1}$ is the inter-user interference from the far user in the same cluster, which will be canceled out with perfect SIC. Thus, $y_{1,1}$ will be reduced to

$$
y_{1,1}=\mathbf{h}_{1,1} \sqrt{d_{1,1}^{-\lambda} P_{1}} \mathbf{w}_{1} \sqrt{\alpha_{1,1}} s_{1,1}+e_{1,1} .
$$

The received SINR of the near user in cluster 1 is then

$$
\begin{aligned}
\operatorname{SINR}_{1,1} & =\frac{\left|\mathbf{h}_{1,1} \sqrt{d_{1,1}^{-\lambda} P_{1}} \mathbf{w}_{1} \sqrt{\alpha_{1,1}}\right|^{2}}{\sigma^{2}}=\frac{\left|\mathbf{h}_{1,1}\right|^{2} d_{1,1}^{-\lambda} \alpha_{1,1} P_{1}}{\sigma^{2}} \\
& =\frac{\chi_{1,1} \alpha_{1,1} d_{1,1}^{-\lambda} P_{1}}{\sigma^{2}}
\end{aligned}
$$

where $\chi_{1,1}$ follows a gamma distribution with $\operatorname{Gamma}(2,1)$. Similarly, the received SINR of the near user in the cluster 2 will be $\operatorname{SINR}_{1,2}=\frac{\chi_{1,2} \alpha_{1,2} d_{1,2}^{-\lambda} P_{2}}{\sigma^{2}}$.

On the other hand, the received signal of the far user in cluster 1 is

$$
\begin{aligned}
y_{2,1}= & \mathbf{h}_{2,1} \sqrt{d_{2,1}^{-\lambda} P_{1}} \mathbf{w}_{1} x_{1}+\mathbf{h}_{2,1} \sqrt{d_{2,1}^{-\lambda} P_{2}} \mathbf{w}_{2} x_{2}+e_{2,1} \\
= & \mathbf{h}_{2,1} \sqrt{d_{2,1}^{-\lambda} P_{1}} \mathbf{w}_{1}\left(\sqrt{\alpha_{1,1}} s_{1,1}+\sqrt{\alpha_{2,1}} s_{2,1}\right) \\
& +\mathbf{h}_{2,1} \sqrt{d_{2,1}^{-\lambda} P_{2}} \mathbf{w}_{2} x_{2}+e_{2,1} .
\end{aligned}
$$

Unlike the near user, the ZFBF vector does not satisfy (3) for the far user in cluster $n$ since we only have one beamforming vector, and the far user does not perform SIC. Therefore, the received SINR of far user in cluster 1 is

$$
\begin{aligned}
& \operatorname{SINR}_{2,1} \\
& =\frac{\left|\mathbf{h}_{2,1} \mathbf{w}_{1}\right|^{2} d_{2,1}^{-\lambda} \alpha_{2,1} P_{1}}{\left|\mathbf{h}_{2,1} \mathbf{w}_{1}\right|^{2} d_{2,1}^{-\lambda} \alpha_{1,1} P_{1}+\left|\mathbf{h}_{2,1} \mathbf{w}_{2}\right|^{2} d_{2,1}^{-\lambda} P_{2}+\sigma^{2}} .
\end{aligned}
$$

The expression of received SINR of the far user in cluster 2 also can be written as

$$
\operatorname{SINR}_{2,2}=\frac{\left|\mathbf{h}_{2,2} \mathbf{w}_{2}\right|^{2} d_{2,2}^{-\lambda} \alpha_{2,2} P_{2}}{\left|\mathbf{h}_{2,2} \mathbf{w}_{2}\right|^{2} d_{2,2}^{-\lambda} \alpha_{1,2} P_{2}+\left|\mathbf{h}_{2,2} \mathbf{w}_{1}\right|^{2} d_{2,2}^{-\lambda} P_{1}+\sigma^{2}},
$$

Since the inter-user interference and inter-cluster interference cannot be removed for the far user, it is required to calculate the distribution of the squared-norm of the product between channel gain and the beamforming vector for further analysis.

\section{Outage Probability Analysis}

We define the outage probability as the probability that the achieved data rate of an individual user is below a predefined value. According to the Shannon capacity and the SINR expressions in (7) and (9), the data rate of the near user and the far user in cluster 1 can be expressed as

$R_{1,1}=\log \left(1+\operatorname{SINR}_{1,1}\right)=\log \left(1+\frac{\chi_{1,1} \alpha_{1,1} d_{1,1}^{-\lambda} P_{1}}{\sigma^{2}}\right)$,

$$
\begin{aligned}
& R_{2,1}=\log \left(1+\operatorname{SINR}_{2,1}\right) \\
& =\log \left(1+\frac{\left|\mathbf{h}_{2,1} \mathbf{w}_{1}\right|^{2} d_{2,1}^{-\lambda} \alpha_{2,1} P_{1}}{\left|\mathbf{h}_{2,1} \mathbf{w}_{1}\right|^{2} d_{2,1}^{-\lambda} \alpha_{1,1} P_{1}+\left|\mathbf{h}_{2,1} \mathbf{w}_{2}\right|^{2} d_{2,1}^{-\lambda} P_{2}+\sigma^{2}}\right) .
\end{aligned}
$$

\section{A. Far user}

The outage probability of the far user can be directly defined as the probability that the data rate falls below the target rate.

$$
\begin{aligned}
& \operatorname{Pr}\left(R_{2,1} \leq \gamma_{2,1}\right)= \\
& \operatorname{Pr}\left(\frac{\left|\mathbf{h}_{2,1} \mathbf{w}_{1}\right|^{2} d_{2,1}^{-\lambda} \alpha_{2,1} P_{1}}{\left|\mathbf{h}_{2,1} \mathbf{w}_{1}\right|^{2} d_{2,1}^{-\lambda} \alpha_{1,1} P_{1}+\left|\mathbf{h}_{2,1} \mathbf{w}_{2}\right|^{2} d_{2,1}^{-\lambda} P_{2}+\sigma^{2}} \leq e^{\gamma_{2,1}}-1\right)
\end{aligned}
$$

Considering high SNR regimes, the noise power is negligible, and hence, the outage probability of the far user in cluster 1 can be approximated by a lower bound ignoring the noise power. Consequently, after some simple mathematical manipulations, the outage probability can be summarized as

$\operatorname{Pr}\left(R_{2,1} \leq \gamma_{2,1}\right) \approx \operatorname{Pr}\left(\frac{\alpha_{2,1} P_{1}}{e^{\gamma_{2,1}}-1}-\alpha_{1,1} P_{1} \leq \frac{\left|\mathbf{h}_{2,1} \mathbf{w}_{2}\right|^{2}}{\left|\mathbf{h}_{2,1} \mathbf{w}_{1}\right|^{2}} P_{2}\right)$.

To derive the closed-form outage expression of the far user, it is necessary to know the ratio distribution between $\left|\mathbf{h}_{2,1} \mathbf{w}_{1}\right|^{2}$ 

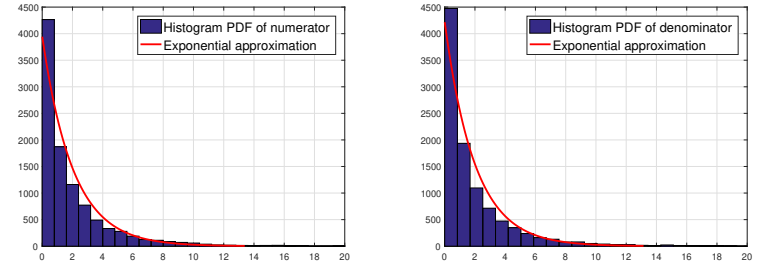
(a)
$\left|\mathbf{h}_{1,2}\right|^{2}\left|h_{2,1}^{2} h_{1,1}^{1}-h_{2,1}^{1} h_{1,1}^{2}\right|^{2}$
$\left|\mathbf{h}_{1,1}\right|^{2}\left|h_{2,1}^{1} h_{1,2}^{2}-h_{2,1}^{2} h_{1,2}^{1}\right|^{2}$

(b)

Fig. 2: Curve fitting method

and $\left|\mathbf{h}_{2,1} \mathbf{w}_{2}\right|^{2}$, where $\mathbf{h}_{2,1}$ is a row vector with complex normal random variables for Rayleigh channels, but the distribution of the column vector $\mathbf{w}_{n}$ is unknown. Hence, we will analyze the expressions, both $\mathbf{h}_{2,1} \mathbf{w}_{1}$ and $\mathbf{h}_{2,1} \mathbf{w}_{2}$ in the following. First, according to the beamforming matrix, the beamforming vectors $\mathbf{w}_{1}$ and $\mathbf{w}_{2}$ can be obtained as

$$
\mathbf{w}_{1}=\left[\begin{array}{c}
\frac{h_{1,2}^{2}\left|\mathbf{h}_{1,1}\right|}{h_{1,1}^{1} h_{1,2}^{2}-h_{1,2}^{1} h_{1,1}^{2}} \\
\frac{-h_{1,2}^{1}\left|\mathbf{h}_{1,1}\right|}{h_{1,1}^{1} h_{1,2}^{2}-h_{1,2}^{1} h_{1,1}^{2}}
\end{array}\right], \mathbf{w}_{2}=\left[\begin{array}{c}
\frac{-h_{1,1}^{2}\left|\mathbf{h}_{1,2}\right|}{h_{1,1}^{1} h_{1,2}^{2}-h_{1,2}^{1} h_{1,1}^{2}} \\
\frac{h_{1,1}^{1}\left|\mathbf{h}_{1,2}\right|}{h_{1,1}^{1} h_{1,2}^{2}-h_{1,2}^{1} h_{1,1}^{2}}
\end{array}\right] .
$$

Then, the ratio between $\left|\mathbf{h}_{2,1} \mathbf{w}_{1}\right|^{2}$ and $\left|\mathbf{h}_{2,1} \mathbf{w}_{1}\right|^{2}$ can be described as

$$
\frac{\left|\mathbf{h}_{2,1} \mathbf{w}_{2}\right|^{2}}{\left|\mathbf{h}_{2,1} \mathbf{w}_{1}\right|^{2}}=\frac{\left|\mathbf{h}_{1,2}\right|^{2}\left|h_{2,1}^{2} h_{1,1}^{1}-h_{2,1}^{1} h_{1,1}^{2}\right|^{2}}{\left|\mathbf{h}_{1,1}\right|^{2}\left|h_{2,1}^{1} h_{1,2}^{2}-h_{2,1}^{2} h_{1,2}^{1}\right|^{2}},
$$

where $h_{1,1}^{1}, h_{1,1}^{2}, h_{1,2}^{1}, h_{1,2}^{2}, h_{2,1}^{1}$ and $h_{2,1}^{2}$ are i.i.d complex, zero mean random normal distributed variables. The distribution of product of two complex normal distributed random variables follows the product normal distribution, however this distribution is not helpful to find tractable expressions for outage probabilities. To calculate (13), we need to find the ratio distribution between $\left|\mathbf{h}_{1,2}\right|^{2}\left|h_{2,1}^{2} h_{1,1}^{1}-h_{2,1}^{1} h_{1,1}^{2}\right|^{2}$ and $\left|\mathbf{h}_{1,1}\right|^{2}\left|h_{2,1}^{1} h_{1,2}^{2}-h_{2,1}^{2} h_{1,2}^{1}\right|^{2}$. Based on the study in [15], the squared norm of complex normal random variables can be approximated as exponential distributed random variables when real and imaginary parts are independent and they have the same variance values. We also verify this by using a curve fitting method as shown in Fig. 2.

It can be clearly observed that the PDFs of numerator and denominator of $\frac{\left|\mathbf{h}_{2,1} \mathbf{w}_{2}\right|^{2}}{\left|\mathbf{h}_{2,1} \mathbf{w}_{1}\right|^{2}}$ can be accurately approximated by an exponential distribution. Therefore, the ratio of two squared norm expressions can be approximated as the ratio between two independent exponential distributions as $\frac{X}{Y}$, where $X \sim$ $\operatorname{Exp}\left(\lambda_{1}\right)$ and $Y \sim \operatorname{Exp}\left(\lambda_{2}\right)$. The corresponding probability density functions (PDF) of $X$ and $Y$ are

$$
\begin{gathered}
f_{X}(x)=\lambda_{1} e^{-\lambda_{1} x}, \\
f_{Y}(y)=\lambda_{2} e^{-\lambda_{2} y} .
\end{gathered}
$$

The main challenge of this step is to derive the PDF and CDF of this ratio distribution. First, assuming $Z=\frac{X}{Y}$ as another random variable representing the ratio of the random variable
$X$ and $Y$. Using the method of transformation [16], the PDF of $Z$ is derived as

$$
\begin{aligned}
& Z=\frac{X}{Y}, \quad X=Z Y, \\
& \frac{d x}{d z}=y, \quad \frac{d x}{d y}=z, \quad \text { and } \quad \frac{d y}{d z}=0 .
\end{aligned}
$$

Then, the Jacobian matrix of transformation is given by $J=\left|\begin{array}{ll}\frac{d x}{d z} & \frac{d x}{d y} \\ \frac{d y}{d z} & \frac{d y}{d y}\end{array}\right|$, hence $J=\left|\begin{array}{ll}y & z \\ 0 & 1\end{array}\right|=y$. According to the properties of joint PDF of $x$ and $y, f_{X, Y}(x, y)$ is derived as $f_{X}(x) f_{Y}(y)=\lambda_{1} \lambda_{2} e^{-\lambda_{1} x+\lambda_{2} y}$, hence for $X=Y Z$, $f_{X, Y}(y z, y)=\lambda_{1} \lambda_{2} e^{-\left(\lambda_{1} z+\lambda_{2}\right) y}$.

Thus, the marginal PDF of random variable $Z$ is given by

$$
\begin{aligned}
& f_{Z}(z)=\int_{-\infty}^{\infty} f(y z, y)|J| d y=\int_{0}^{\infty} f(y z, y)|J| d y \\
& =\lambda_{1} \lambda_{2} \int_{0}^{\infty} e^{-\left(\lambda_{1} z+\lambda_{2}\right) y} y d y .
\end{aligned}
$$

Let $\theta=\lambda_{1} z+\lambda_{2}$,

$$
f_{Z}(z)=\lambda_{1} \lambda_{2} \int_{0}^{\infty} y e^{-\theta y} d y
$$

By definition, $\mathbf{E}(z)=\int_{0}^{\infty} \theta z e^{-\theta z} d z=\frac{1}{\theta}$ which is the mean of an exponential distribution with parameter $\theta, f_{Z}(z)=$ $\frac{\lambda_{1} \lambda_{2}}{\theta^{2}}$. Therefore, the PDF of the ratio distribution is

$$
f_{Z}(z)=\frac{\lambda_{1} \lambda_{2}}{\left(\lambda_{1} z+\lambda_{2}\right)^{2}}
$$

The CDF of the ratio distribution will be

$$
F_{Z}(z)=\int_{0}^{z} f(t) d t=\int_{0}^{z} \frac{\lambda_{1} \lambda_{2}}{\left(\lambda_{1} t+\lambda_{2}\right)^{2}} d t=\frac{\lambda_{1} z}{\lambda_{1} z+\lambda_{2}} .
$$

According to the PDF and CDF of the ratio distribution, the closed-form outage expression is derived as

$$
\begin{aligned}
& \operatorname{Pr}\left(R_{2,1} \leq \gamma_{2,1}\right)=\operatorname{Pr}\left(\operatorname{SINR}_{2,1} \leq e^{\gamma_{2,1}}-1\right) \\
& =1-\operatorname{Pr}\left(\frac{\left|\mathbf{h}_{2,1} \mathbf{w}_{2}\right|^{2}}{\left|\mathbf{h}_{2,1} \mathbf{w}_{1}\right|^{2}} \leq \frac{\alpha_{2,1} P_{1}}{\left(e^{\gamma_{2,1}}-1\right) P_{2}}-\frac{\alpha_{1,1} P_{1}}{P_{2}}\right) \\
& =1-\frac{\lambda_{1}}{\lambda_{1}+\frac{\lambda_{2}}{\left(\frac{\alpha_{2,1} P_{1}}{\left(e^{\gamma_{2}, 1}-1\right) P_{2}}-\frac{\alpha_{1,1} P_{1}}{P_{2}}\right)}} .
\end{aligned}
$$

Subsequently, based on the condition of transmit power allocation coefficient that $\alpha_{2,1}=1-\alpha_{1,1}$ and introducing a new variable, power ratio coefficient $\rho=\frac{P_{1}}{P_{2}}$, we have

$$
\operatorname{Pr}\left(R_{2,1} \leq \gamma_{2,1}\right)=1-\frac{\lambda_{1}}{\lambda_{1}+\frac{\lambda_{2}\left(e^{\gamma_{2,1}}-1\right)}{\rho\left(1-\alpha_{1,1} e^{\gamma_{2}, 1}\right)}},
$$

where $\lambda_{1}=\lambda_{2}$, since $\left|\mathbf{h}_{1,2}\right|^{2}\left|h_{2,1}^{2} h_{1,1}^{1}-h_{2,1}^{1} h_{1,1}^{2}\right|^{2}$ and $\left|\mathbf{h}_{1,1}\right|^{2}\left|h_{2,1}^{1} h_{1,2}^{2}-h_{2,1}^{2} h_{1,2}^{1}\right|^{2}$ have the same distribution.

Therefore, the final outage expression of the far user in cluster 1 will be

$$
\operatorname{Pr}\left(R_{2,1} \leq \gamma_{2,1}\right)=\frac{e^{\gamma_{2,1}}-1}{e^{\gamma_{2,1}}\left(1-\alpha_{1,1} \rho\right)-(1-\rho)} .
$$

The outage probability of the far user in cluster 2 has the

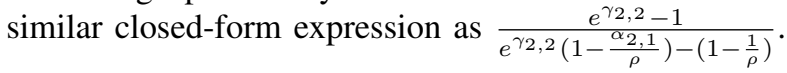




\section{B. Near user}

As SIC is performed at the near user, the outage probability of the near user also depends on the successful SIC of the far user's signal. Therefore, the outage probability of the near user in cluster $n$ can be defined as

$$
\begin{gathered}
\operatorname{Pr}_{1, n}^{\text {out }}=1-\operatorname{Pr}_{1, n}^{\mathrm{suc}} \times \operatorname{Pr}_{2, n}^{\mathrm{suc}} . \\
(n=1,2)
\end{gathered}
$$

First, the success probability of the near user can be obtained based on the cumulative distribution function (CDF) of an exponential random variable, which is represented as

$$
\begin{aligned}
\operatorname{Pr}_{1, n}^{\mathrm{suc}}\left(R_{1, n} \geq \gamma_{1, n}\right) & =\operatorname{Pr}\left(\chi_{1, n} \geq \frac{\left(e^{\gamma_{1, n}}-1\right) \sigma^{2}}{\alpha_{1, n} d_{1, n}^{-\lambda} P_{n}}\right) \\
& =1-\gamma\left(2, \frac{\left(e^{\gamma_{1, n}}-1\right) \sigma^{2}}{\alpha_{1, n} d_{1, n}^{-\lambda} P_{n}}\right)
\end{aligned}
$$

where $\gamma_{1, n}$ is the minimum reserved rate, and $P_{n}$ is the transmit power in cluster $n$.

Then, the probability of successful detection of the far user's signal by the near user can be formulated as

$$
\begin{aligned}
& \operatorname{Pr}_{2, n}^{\text {suc }}\left(R_{2, n}^{\prime} \geq \gamma_{2, n}^{\prime}\right) \\
& = \begin{cases}1-\gamma\left(2, \frac{\left(e^{\gamma_{2, n}^{\prime}}-1\right) \sigma^{2}}{d_{1, n}^{-\lambda} P_{n}\left(1-\alpha_{1, n} e^{\gamma_{2, n}^{\prime}}\right)}\right), & f \geq 0 \\
0, & f<0\end{cases}
\end{aligned}
$$

where $R_{2, n}^{\prime}=\log \left(1+\frac{\left|\mathbf{h}_{1, n}\right|^{2} d_{1, n}^{-\lambda} \alpha_{2, n} P_{n}}{\left|\mathbf{h}_{1, n}\right|^{2} d_{1, n}^{-\lambda} \alpha_{1, n} P_{n}+\sigma^{2}}\right)$ and $f=1-$ $e^{\gamma_{2, n}^{\prime}} \alpha_{1, n}$. Finally, the outage probability of the near user in cluster $n$ can be obtained.

\section{Simulation Results}

To validate the accuracy of the analytical outage expressions, Monte Carlo simulations are provided to assess the precision of the closed-form outage expressions for both users. The transmit power allocation coefficient $\alpha_{1,1}$ is smaller than $\alpha_{1,2}$ due to the properties of power control for downlink NOMA system. The value of the transmit power for cluster 1 is fixed to $P_{1}=10$.

Fig. 3 shows the relationship between the outage probability of the far user in cluster 1 and the target rate. The power ratio coefficient $\rho=\frac{P_{1}}{P_{2}}$ acts a key factor in the closed-form outage expression for far user. Thus, we consider two cases, $\rho=1$ and $\rho=10$. For $\rho=1$ the power levels of two clusters are the same which also implies that both clusters are at the same distance from BS, while $\rho=10$ represents a case where the power level of cluster 1 is larger than the one for cluster 2. We also assume that $d_{2,1}=10$ and $d_{1,1}=1$ unless otherwise stated. It is observed that the outage probabilities of far user increase with the target rate. For comparison, the outage probability with same power level $\rho=1$ is greater than in the case of $\rho=10$. It is also shown that the analytical results match well with the numerical results.

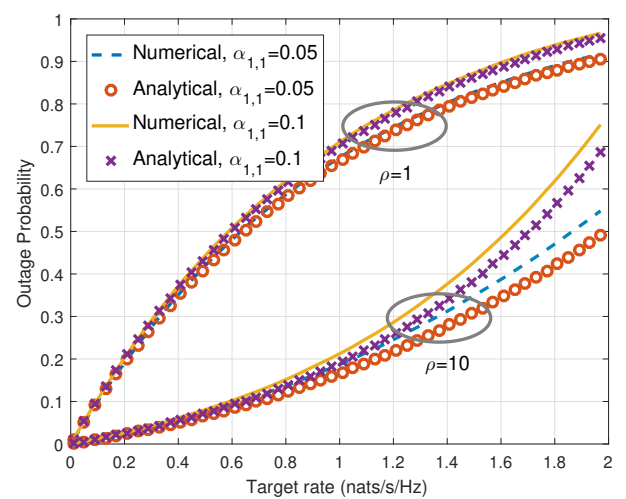

Fig. 3: The outage probability of the far user vs target rate with different $\alpha_{1,1}$

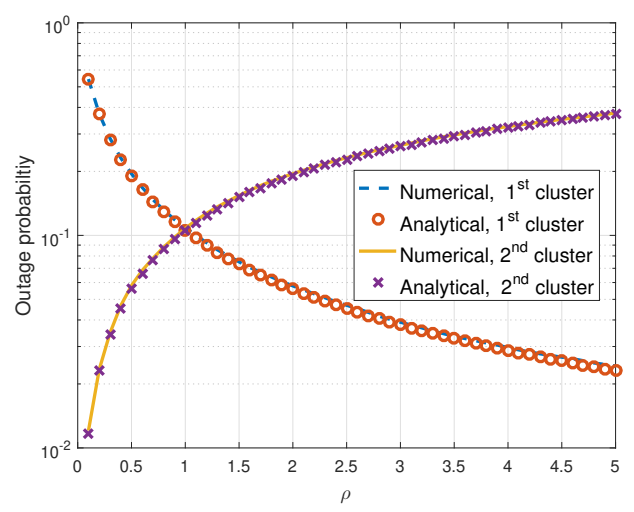

Fig. 4: The outage probability of the far user of both clusters

To further study the effects of power ratio $\rho$ on outage probabilities, in Fig. 4, we compare the outage probabilities of far users for both clusters. We observe that the outage probability decreases with $\rho$ for cluster 1 , but it increases with $\rho$ for cluster 2. In particular, the outage probabilities cross together when power level is the same (i.e., $\rho=1$ ). It also confirms that the analytical results for outage probabilities have as high accuracy compared with the numerical results.

Fig. 5 illustrates the outage probability of the near user versus target rate with various values of $\alpha_{1, n}$. The SNR of cluster $n$ is fixed to 20dB. As can be observed, the outage probability of the near user improves as the target rate increases. Moreover, the outage probability decreases with the growth of $\alpha_{1, n}$ and the outage performance becomes worse as the distance of the near user from BS increases.

Fig. 6 demonstrates the effects of SNR (which is defined as $\left.P_{n} / \sigma^{2}\right)$ on the outage probability of the near user in cluster $n$. For $\gamma_{1, n}=1$, we can see that the outage probability decreases with the SNR. Moreover, the results indicate that the outage probability of the near user decreases with the growth of $\alpha_{1, n}$ since the allocated power to the far user becomes smaller. In addition, the outage probability increases with the distance from BS to near user. The outage performance of 


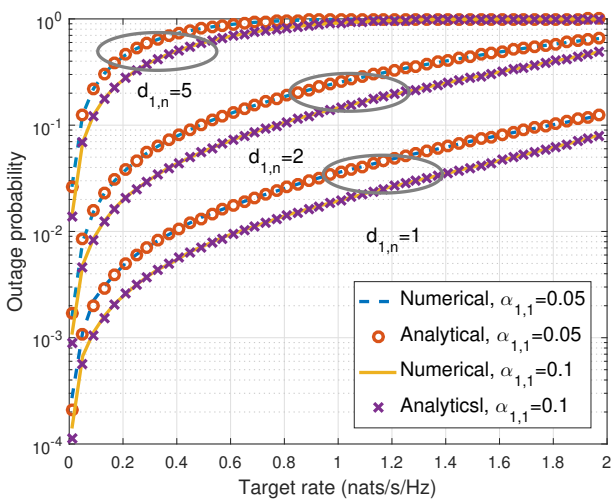

Fig. 5: The outage probability of the near user vs target rate with different $\alpha_{1, n}(\mathrm{SNR}=20 \mathrm{~dB})$

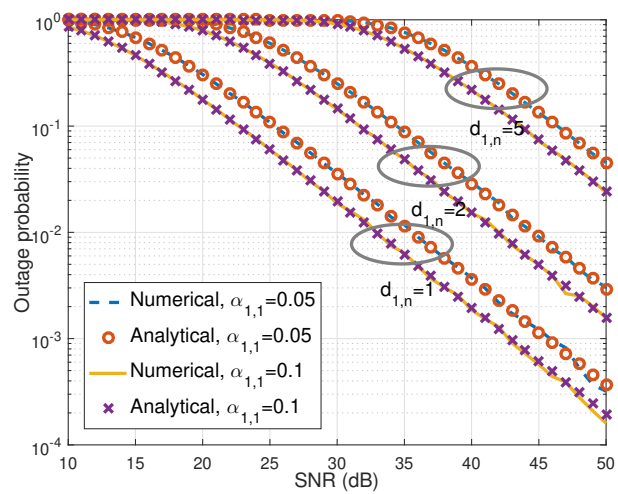

Fig. 6: The outage probability of the near user vs SNR with different $\alpha_{1, n}\left(\gamma_{1, n}=1\right)$

the near user has a fine agreement between the numerical and analytical results, and verifies the validity of our outage probability analysis.

\section{CONCLUSIONS}

In this paper, the closed-form outage expressions of individual users have been derived for downlink MISO-NOMA system in case of two antennas. The outage probability of far users can be approximated as the CDF of the ratio of two exponentially distributed random variables, which can be solved by applying Jacobian transformation method. On the other hand, the outage probability of the near users for both clusters is proven to be in the form of the CDF of an exponential distribution. This work is the first step to derive tractable expressions for the outage probability of MISO NOMA systems under assumption of statistical CSI and hence it only investigates the case of two antennas. In the future, we will extend this work for a general case of $N$ antennas.

\section{ACKNOWLEDGEMENT}

The authors would like to thank Jakob Hoydis (Bell Lab) and Prof Francois Baccellii (INRIA) for their helpful discussion and comments.

\section{REFERENCES}

[1] L. Dai, B. Wang, Y. Yuan, S. Han, C. I, and Z. Wang, "Non-orthogonal multiple access for 5G: Solutions, challenges, opportunities, and future research trends," IEEE Communications Magazine, vol. 53, no. 9, pp. 74-81, Sep. 2015.

[2] Y. Liu, H. Xing, C. Pan, A. Nallanathan, M. Elkashlan, and L. Hanzo, "Multiple-antenna-assisted non-orthogonal multiple access," IEEE Wireless Communications, vol. 25, no. 2, pp. 17-23, Apr. 2018.

[3] B. Kimy, S. Lim, H. Kim, S. Suh, J. Kwun, S. Choi, C. Lee, S. Lee, and D. Hong, "Non-orthogonal multiple access in a downlink multiuser beamforming system," in IEEE Military Communications Conference, Nov. 2013.

[4] Y. Liu, G. Pan, H. Zhang, and M. Song, "On the capacity comparison between MIMO-NOMA and MIMO-OMA," IEEE Access, vol. 4, pp. 2123-2129, 2016.

[5] M. F. Hanif, Z. Ding, T. Ratnarajah, and G. K. Karagiannidis, "A minorization-maximization method for optimizing sum rate in the downlink of non-orthogonal multiple access systems," IEEE Transactions on Signal Processing, vol. 64, no. 1, pp. 76-88, 2016.

[6] Z. Liu, L. Lei, N. Zhang, G. Kang, and S. Chatzinotas, "Joint beamforming and power optimization with iterative user clustering for MISONOMA systems," IEEE Access, vol. 5, pp. 6872-6884, May 2017.

[7] Y. Sun, J. Zhou, Q. Cao, and S. Li, "Precoder design in statistical CSI aided non-orthogonal multiple access," IEEE Access, vol. 6, pp. 16484$16492,2018$.

[8] S. Mao, S. Leng, J. Hu, and K. Yang, "Power minimization resource allocation for underlay MISO-NOMA SWIPT systems," IEEE Access, vol. 7, pp. 17247-17 255, 2019

[9] Y. Liu, M. Elkashlan, Z. Ding, and G. K. Karagiannidis, "Fairness of user clustering in MIMO non-orthogonal multiple access systems," IEEE Communications Letters, vol. 20, no. 7, pp. 1465-1468, Jul. 2016.

[10] Y. Liu, M. Derakhshani, and S. Lambotharan, "Outage analysis and power allocation in uplink non-orthogonal multiple access systems," IEEE Communications Letters, vol. 22, no. 2, pp. 336-339, 2017.

[11] S. Li, M. Derakhshani, and S. Lambotharan, "Outage-constrained robust power allocation for downlink MC-NOMA with imperfect SIC," in IEEE International Conference on Communications (ICC), 2018, pp. $1-7$

[12] F. Cui, Z. Qin, Y. Cai, M. Zhao, and G. Y. Li, "Rethinking outage constraints for resource management in NOMA networks," IEEE Journal of Selected Topics in Signal Processing, vol. 13, no. 3, pp. 423-435, June 2019.

[13] J. Cui, Z. Ding, and P. Fan, "Outage probability constrained MIMONOMA designs under imperfect CSI," IEEE Transactions on Wireless Communications, vol. 17, no. 12, pp. 8239-8255, Dec. 2018.

[14] N. Jindal and A. Goldsmith, "Dirty-paper coding versus TDMA for MIMO broadcast channels," IEEE Transactions on Information Theory, vol. 51, no. 5, pp. 1783-1794, May 2005.

[15] J. M. Romero-Jerez and F. J. Lopez-Martinez, "On the distribution of the squared norm of non-circular complex gaussian random variables with applications," in IEEE International Symposium on Information Theory (ISIT), Jun. 2015, pp. 341-345.

[16] A. M. Mathai, Jacobians of matrix transformations and functions of matrix arguments. World Scientific Publishing Company, 1997. 